\title{
Pathological Changes of Urinary System in a Dog with Urolithiasis and Renal Papillary Necrosis
}

\author{
Tuncer Kutlu', Gözde Yücel-Tenekeci², Mert Pekcan ${ }^{3}$, Emir Yengi Kule ${ }^{4}$ \& Osman Kutsal²
}

\begin{abstract}
Background: Urolithiasis is one of the important lower urinary tract diseases in dogs. Uroliths develop when urine becomes "supersaturated". Struvite urolithiasis in dogs is formed when urine saturated with magnesium, ammonium and phosphate. Renal papillary necrosis is a form of nephropathy involving the necrosis of the renal papilla. The aim of this case is the evaluation of histopathological changes in kidney and urinary bladder caused by urolithiasis with sitruvite, a pathology commonly seen in dogs. Renal papillary necrosis that encountered in the case was evaluated histopathologically. We intend to discuss the relationship between urolithiasis and renal papillary necrosis.

Case: The material of the case was a 7.5 year-old Rottweiler female dog that had been operated for the urolithiasis treatment and died after surgery. The animal sent to pathology department was necropsied and evaluated histopathologically. Also the uroliths removed during the surgery were sent for analysis postoperatively. Qualitative composition of uroliths was determined by a series of chemical analyses in Department of Biochemistry. X-ray diffraction was performed as well in General Directorate of Mineral Research and Exploration in the objective of result verification. Macroscopically, 30 pieces uroliths with varying size from $1 \mathrm{~mm}$ to $3 \mathrm{~cm}$ were seen in urinary bladder. They were $110 \mathrm{~g}$ in weight with smooth surface and round and/or ovoid shapes. After necropsy, specimens from kidney and urinary bladder were fixed in $10 \%$ neutral buffered formalin, embedded in paraffin wax, cut at $5 \mu \mathrm{m}$, and stained with hematoxylin and eosin (HE). Bladder wall thickening and hemorrhage on mucosa were noticed. Kidneys had roughened surface with the capsule being difficult to peel off. Also, renal papillary necrosis, reddish in color was observed in papilla of the right kidney. Microscopically, crystal formations were observed proximal tubule lumens of kidneys. Interstitial nephritis in cortex and medulla had been noticed in both kidneys. Interstitial nephritis has been noticed in both kidneys. Hemorrhagic necrotic area demarcated by connective tissue was encountered in medulla of the right kidney. In the lamina propria of urinary bladder hemorrhage were noted. Desquamation and degeneration of lamina epithelialis and wide hemorrhage area in lamina propria were noted in urinary bladder. Results obtained from biochemical analysis were verified with x-ray diffraction method, uroliths were determined as magnesium ammonium phosphate (struvite).

Discussion: Renal papillary necrosis is a form of nephropathy involving the necrosis of the renal papilla, caused by ischemia of the inner medulla of the kidney. According to the first report of renal papillary necrosis, symmetrical soft calyceal concretions and their appearances are typical lesions for diagnosis of renal papillary necrosis. The presence of crystals in the tubule lumen and the formation of struvite stone (which has the same chemical composition with calyceal concretions) established a distinct relationship between urolithiasis and renal papillary necrosis. This case showed that struvite stones can reach large volumes in urinary bladder, and caused bladder wall thickness and hemorrhages. Also, the lower urinary tract obstruction or urolithiasis should be remembered in the renal papillary necrosis together with the risk of hydronephrosis.
\end{abstract}

Keywords: dog, histopathology, renal papillary necrosis, struvite, urolithiasis 


\section{INTRODUCTION}

Urolithiasis is one of the important lower urinary tract diseases in dogs. Uroliths develop when urine becomes "supersaturated" [7]. One of the more common urolith in the dog is struvite. Struvite urolithiasis in dogs is formed when urine saturated with magnesium, ammonium and phosphate $[3,11,12]$. This saturation of urine is usually caused by urinary tract infections with urease-producing bacteria like Staphylococcus spp. and Proteus spp. [10,12]. More than $95 \%$ of urolith formation is thought to be related to these infections [3]. Also, female, spayed and small breed dogs are predisposed to struvite urolithiasis [10].

Renal papillary necrosis is a form of nephropathy involving the necrosis of the renal papilla, caused by ischemia of the inner medulla of the kidney $[1,5]$. Papillary necrosis may occur as a primary or secondary lesion. The primary papillary necrosis is caused by prolonged and excessive consumption of nonsteroidal anti-inflammatory drug. Seconder papillary necrosis is related to interstitial fibrosis, reduction in vasa recta blood flow, resulting in reduced blood flow like amyloidosis and hyalinization, and the elevated pressure due to pelvic calculus, vesicoureteral reflux and lower urinary tract obstructions [1]. Pyelonephritis, diabetes mellitus, liver cirrhosis, urinary tract infection are other causes [2,5,8]. In humans approximately $30 \%$ of all cases of renal papillary necrosis occur due to diabetes mellitus [6].

The purpose of the study is to describe pathological changes of urinary system in a Rottweiler dog with urolithiasis and renal papillary necrosis.

\section{CASE}

The material of the case was a 7.5 year-old Rottweiler female dog that had been operated for the urolithiasis treatment and died after surgery. The animal sent to pathology department was necropsied. After necropsy, specimens from kidney and urinary bladder were fixed in $10 \%$ neutral buffered formalin, embedded in paraffin wax, cut at $5 \mu \mathrm{m}$, and stained with hematoxylin and eosin (HE). Also the uroliths removed during the surgery were sent for analysis. Qualitative composition of uroliths was determined by a series of chemical analysis in Department of Biochemistry. X-ray diffraction was performed as well in General Directorate of Mineral Research and Exploration in the objective of result verification.
Macroscopically, 30 pieces uroliths with varying size from $1 \mathrm{~mm}$ to $3 \mathrm{~cm}$ were seen in urinary bladder (Figure 1A). They were $110 \mathrm{~g}$ in weight with smooth surface and round and/or ovoid shapes. Bladder wall thickening and hemorrhage were noticed. Kidneys had roughened surface with the capsule being difficult to peel off. Also, renal papillary necrosis, reddish in color was observed in papilla of the right kidney (Figure 1B).

Microscopically, crystal formations were observed proximal tubule lumen and interstitial nephritis in cortex and medulla had been noticed in both kidneys (Figure 2A,B). Also, hemorrhagic necrotic area demarcated by connective tissue was encountered in medulla of the right kidney (Figure 2C). Desquamation and degeneration of lamina epithelialis and wide hemorrhage area in lamina propria were noted in urinary bladder (Figure 2D). Results obtained from biochemical analysis were verified with $\mathrm{x}$-ray diffraction method, uroliths were determined as magnesium ammonium phosphate (struvite).

\section{DISCUSSION}

According to Dr. Johann Wagner who performed autopsy of Ludwig van Beethoven (Beethoven's case appears to be the first report of renal papillary necrosis), symmetrical soft calyceal concretions (containing mainly ammonium magnesium phosphate) and their appearances are typical lesions for diagnosis of renal papillar necrosis $[4,6]$. Although the mentioned typical concretions of papilla were not macroscopically observed in our case, the presence of crystals in the tubule lumen and the formation of struvite stone (which has the same chemical composition with calyceal concretions) established a distinct relationship between urolithiasis and renal papillary necrosis.

Renal papillary necrosis in humans has been reported to be seen mostly in diabetic patients. Also, it is a known fact that diabetic patients are more predisposed to urolithiasis because of the association between insulin resistance and defects in renal ammonium production [9]. In addition, although misuse of drugs such as nonsteroidal anti-inflammatory drugs is highly effective in the formation of primer renal papillary necrosis, the most plausible cause for this case is obstruction and vesicoureteral reflux caused by numerous stones in the urinary tract. Unfortunately, 
T. Kutlu, G. Yücel-Tenekeci, M. Pekcan, E.Y. Kule \& O. Kutsal. 2019. Pathological Changes of Urinary System in a Dog with Urolithiasis and Renal Papillary Necrosis. Acta Scientiae Veterinariae. 47(Suppl 1): 420.

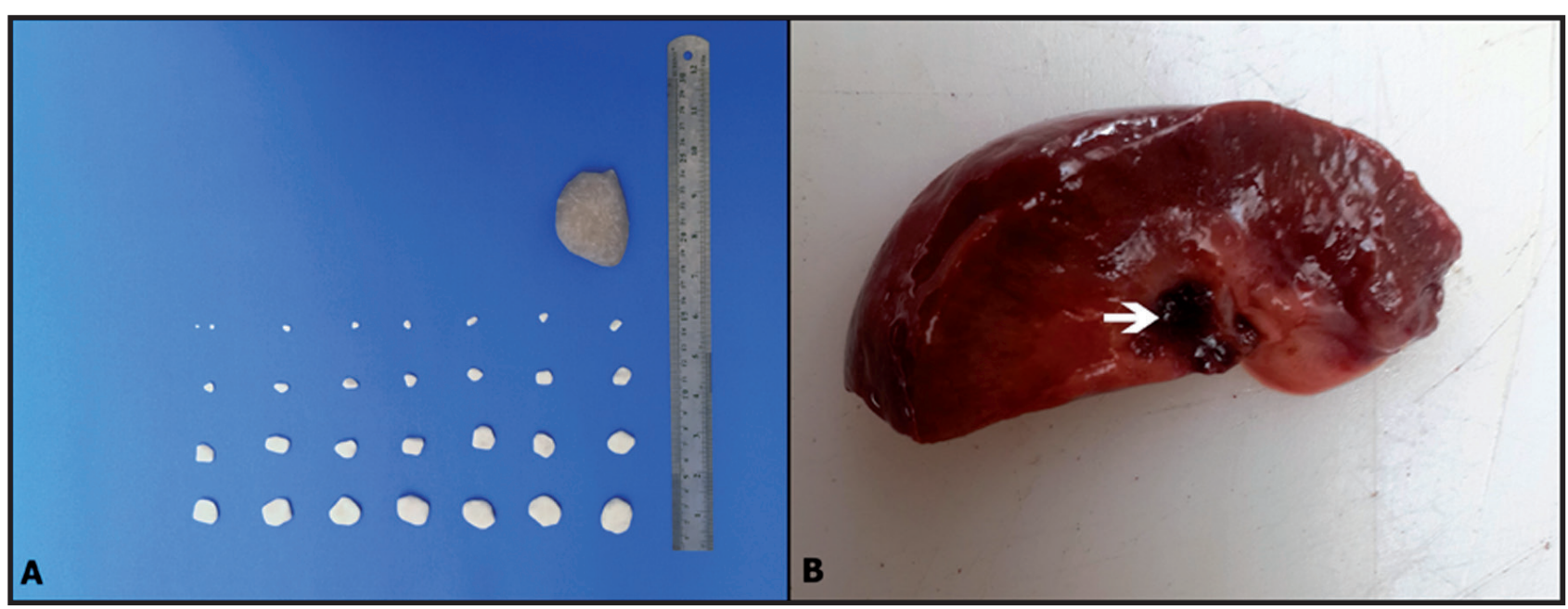

Figure 1. Uroliths and renal papillary necrosis A- Thirty pieces uroliths with varying size from urinary bladder. B- Macroscopical appearance of renal papillary necrosis (arrow).

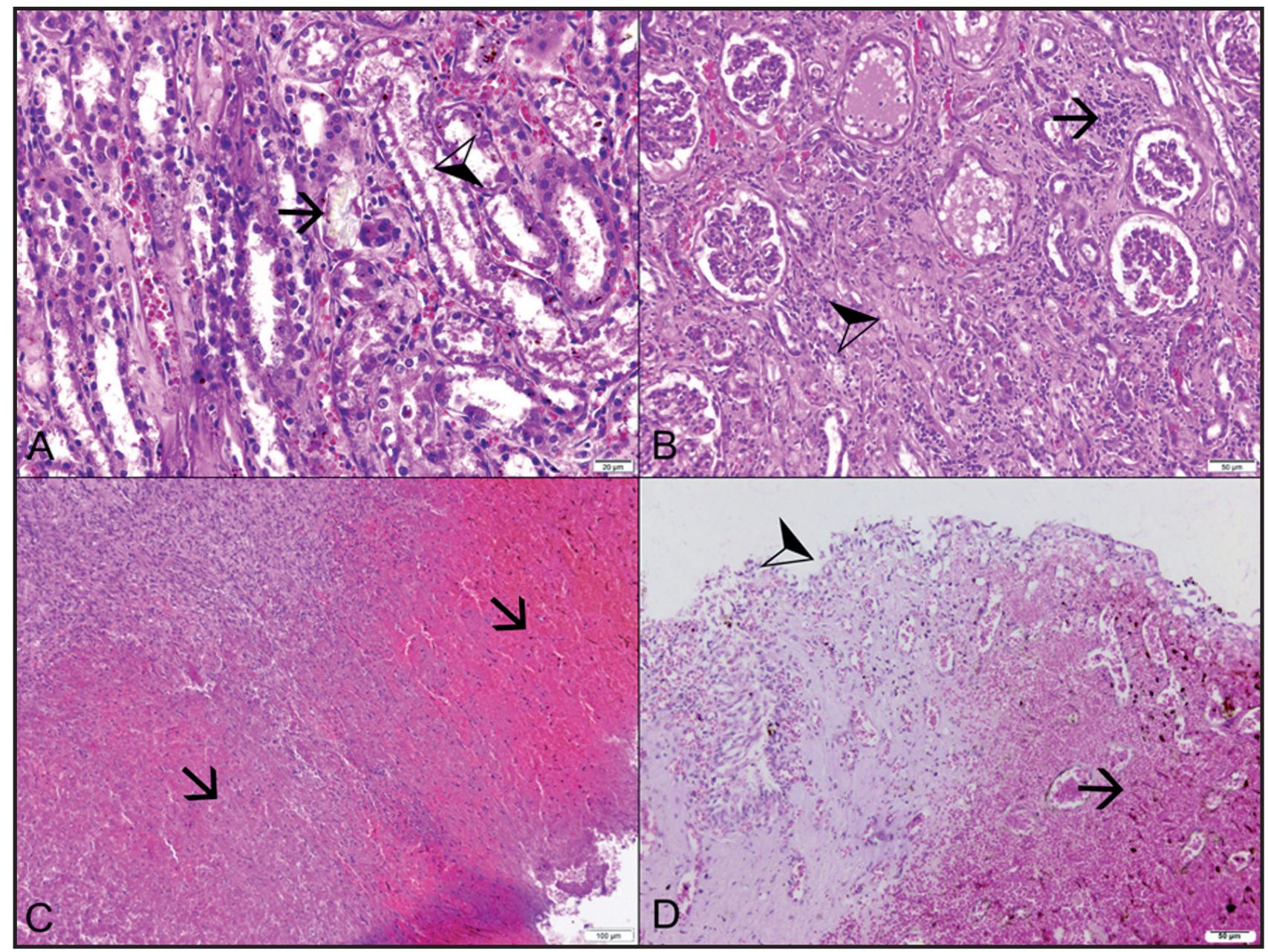

Figure 2. Microscopic findings in urinary system. A- Crystal formation in kidney tubule lumen (arrow), degeneration in tubul epithelium (arrowhead) [HE]. B- Chronic nephritis; mononuclear cell infiltration (arrow), connective tissue proliferation (arrowhead) [HE]. C- Renal papillary necrosis; necrosis and haemorrhage area (arrows) [HE]. D- Desquamation of lamina epithelialis (arrowhead) and wide haemorrhage area in urinary bladder (arrow) [HE].

the biochemical blood profile and the treatment information of the animal were not obtained.

This case showed that struvite stones can reach large volumes in urinary bladder, and caused bladder wall thickness and hemorrhages. Also, the lower urinary tract obstruction or urolithiasis should be remembered in the renal papillary necrosis together with the risk of hydronephrosis. 


\section{REFERENCES}

1 Breshears M.A. \& Confer A.W. 2007. The urinary system. In: Zachary J.F. (Ed). Pathologic Basis of Veterinary. 6th edn. St. Louis: Saunders, pp.617-661.

2 Brix A.E. 2002. Renal papillary necrosis. Toxicologic Pathology. 30: 672-674

3 Chew D.J., Dibartola S.P. \& Schenck P. 2011. Canine and Feline Nephrology and Urology. 2nd edn. St. Louis: Elsevier, pp.275-277.

4 Davies P.J. 1999. Beethoven's nephropathy and death: discussion paper. Journal of the Royal Society of Medicine. 86: $159-161$.

5 Eknoyan C., Qunibi W.Y., Grissom R.T., Tuma S.N. \& Ayus J.C. 1982. Renal papillary necrosis: an update. Medicine. 61: 55-73.

6 Geller S.A. \& Campos F.P.F. 2013. Renal papillary necrosis. Autopsy Case Reports. 3: 69-71.

7 Hoxha Z. \& Rapti D. 2017. Urolithiasis in dogs. Albanian Journal of Agricultural Sciences. (Special edition): 637-640.

8 Jung D.C., Kim S.H. \& Jung S.I. 2006. Renal papillary necrosis: Review and comparison of findings at Multi-Detector Row CT and intravenous urography. Radiographics. 26: 1827-1836.

9 Nerli R., Jali M., Kumar Guntaka A., Patne P., Patil S. \& Hiremath M.B. 2015. Type 2 diabetes mellitus and renal stones. Advanced Biomedical Research. 4: 180.

10 Okafor C.C., Pearl D.L. \& Lefebvre S.L. 2013. Risk factors associated with struvite urolithiasis in dogs evaluated at general care veterinary hospitals in the United States. Journal of the American Veterinary Medical Association. 243: 1737-1745.

11 Palma D., Langstone C., Gisselman K. \& McCue J. 2013. Canine struvite urolithiasis. Compendium: Continuing Education for Veterinarians. 35: E1.

12 Seaman R. \& Bartges J. 2001. Canine struvite urolithiasis. Journal of Exotic Pet Medicine. 23(5): 407-419. 